\title{
Situação do sistema de unidades de conservação do estado de Mato Grosso
}

\author{
Status of conservation units system of Mato Grosso state
}

Henrique Figueiredo da Rocha ${ }^{1}$, Jhonny Pinto Vieira Mendes Moura ${ }^{2}$, Rafael Taques Padilha ${ }^{1}$ e José Guilherme Roquette ${ }^{3}$

${ }^{1}$ Engenheiro Florestal. rocha.henriquef@gmail.com;rafaeltp_8@hotmail.com

${ }^{2}$ Mestrando, Programa de Pós-Graduação em Ciências Florestais e Ambientais, Universidade Federal de Mato Grosso, Cuiabá, Mato Grosso, Brasil.

${ }^{3}$ Mestre, Ministério Público do Estado de Mato Grosso, Cuiabá, Mato Grosso, Brasil.

jose.roquette@mpmt.mp.br

\begin{abstract}
Resumo
O objetivo do trabalho é descrever a situação atual do Sistema Nacional de Unidades de Conservação da Natureza (SNUC), apontar os problemas da gestão e descumprimento a legislação vigente. Com uma estrutura de administração participativa, a rede de informações do SNUC sofre com a falta de comunicação entre os diferentes órgãos. Aliado a desatualização dos domínios oficiais, o sistema falha onde deveria prover dados das unidades de conservação (UC's). O Ministério do Meio Ambiente, responsável por compilar dados referentes a todas unidades de conservação possui um grande déficit de informações referentes as UC's do estado de Mato Grosso, 46 no total. Na base de dados do ICMBio 14 unidades federais são omitidas. Quanto ao plano de manejo, 35 unidades estaduais e 17 unidades federais não possuem o documento. Independentemente da crítica situação das UC's no estado, referente a falta de planos de manejo, as mesmas podem gerar benefícios, como a conservação da biodiversidade local.
\end{abstract}

Palavras-chave: SNUC; Preservação Ambiental; Conservação da Natureza.

\begin{abstract}
The paper's objective is describe the current situation of the National Protected Areas System (SNUC), point out the problems of management and non-compliance with current legislation. With a participatory management structure, the SNUC's network information suffers from a lack of communication between the different agencies. Allied to the outdated official domain, the system fails when should provide data for conservation units (UC's). The Ministry of the Environment, responsible for compiling data of all protected areas has a large deficit of information regarding the UC's of Mato Grosso, 46 in total. In the database of ICMBio, 14 federal units are omitted. As for the management plan, 35 state units and 17 federal units do not have the document. Regardless of the critical situation of UC's in the state, they can generate benefits such as conservation of local biodiversity.
\end{abstract}

Keywords: National Protected Areas System; Environmental Preservation; Nature Conservation. 


\section{Introdução}

Segundo a Lei no 9.985 de 18 de julho de 2000, unidade de conservação é definida como um espaço e seus recursos ambientais, com características relevantes, legalmente instituído pelo Poder Público, com objetivo principal de conservação e preservação dos recursos ambientais. Segundo Terborgh e Van Schaik (2002) são de extrema importância para a manutenção da biodiversidade de uma determinada área de interesse. As unidades de conservação (UC's), em geral, tem característica básica de proteger a biodiversidade local, promover alternativas de uso sustentável dos recursos naturais com atividades de recreação, lazer e ecoturismo, bem como estimular pesquisas científicas (ARAUJO et al., 2011).

Inicialmente, inúmeras unidades foram criadas sem que houvesse um planejamento prévio, estudo que estabelecesse se determinada área possuía relevância para proteção, considerando-se apenas características visuais, e ainda utilizando as unidades de conservação como ferramentas políticas. A constituição deliberada de unidades de conservação pode gerar ineficiência na gestão e controle das mesmas, motivando problemas de ordem fundiária, de recursos humanos e financeiros, de situação política e administrativa, entre outros (BELINASSI et al., 2011).

Para se analisar uma área candidata a unidade de conservação deve-se avaliar sua estrutura de forma geral. Todos os elementos de uma região estão interconectados, não podendo assim proceder a análise de um fragmento do ambiente natural dissociado da região de seu entorno.

O estado de Mato Grosso possui 106 unidades de conservação, ocupando aproximadamente $6 \%$ de sua área total. Apesar de ser o terceiro maior estado em extensão territorial, o mesmo apresenta apenas 23 UC's federais, correspondendo a 6,39\% das unidades dessa instância no Brasil (SEMA, 2015).

O Sistema Nacional de Unidades de Conservação da Natureza (SNUC) foi instituído pela Lei no 9.985 de 18 de julho de 2000, com o objetivo de unificar informações referentes as unidades de conservação em suas três diferentes instâncias (municipal, estadual e federal), contribuir para proteção de espécies ameaçadas de extinção, paisagens, recursos hídricos, edáficos entre outros (BRASIL, 2008).

Segundo Teixeira (2005) o SNUC é o resultado de uma década de discussão entre ambientalistas, cientistas, ONGs, representantes de populações tradicionais, organizações ambientalistas internacionais, organizações privadas, entre outros.

Com diferentes categorias de uso; (doze no total), que são divididas em dois grupos (proteção integral e uso sustentável), cada unidade de conservação, com base em sua biodiversidade de fauna e flora, localização e estágio de degradação, deve ter o uso adequado às suas particularidades.

O Instituto Chico Mendes de Conservação da Biodi- versidade (ICMBIO) foi criado no ano de 2007, pela Lei $\mathrm{n}$ ⒒ 516 de 28 de agosto do respectivo ano. O instituto que é vinculado ao Ministério do Meio Ambiente (MMA) foi desenvolvido como ferramenta para executar as políticas nacionais de unidades de conservação, relacionadas ao uso sustentável de recursos naturais, mas principalmente realizar a articulação entre os demais órgãos e entidades envolvidas (BRASIL, 2007).

Os órgãos responsáveis pela gestão das unidades de conservação podem ser segmentados em consultivo e deliberativo, central e executor, assim, o CONAMA, Conselho Nacional do Meio Ambiente, acompanha e implementa o SNUC, o MMA coordena o sistema e o ICMBIO, associado aos órgãos estaduais e municipais de meio ambiente, analisam a proposta de criação e administração de unidades de conservação, respectivamente (BRASIL, 2000).

Devido a forma de gestão participativa, com a ação de diferentes órgãos, existem problemas de comunicação entre os mesmos, como a falta de atualização e de informações sobre determinadas unidades. Outro problema relevante é o não cumprimento do prazo legal para realizar o plano de manejo da unidade e a organização do conselho gestor. Desse modo, o presente trabalho tem como objetivo descrever os principais problemas da estrutura de gestão e o panorama das unidades de conservação do estado de Mato Grosso.

\section{Material e métodos}

A coleta de informações foi realizada a partir de pesquisa em domínios oficiais para a obtenção de dados públicos referentes às UC's disponíveis no Ministério do Meio Ambiente, Instituto Chico Mendes de Conservação da Biodiversidade e na Secretaria Estadual de Meio Ambiente do Estado de Mato Grosso (SEMA).

A caracterização das UC's segue a classificação descrita no Capítulo IV da Lei no 9.985/2000. Das quais inicialmente são divididas em unidades de proteção integral e unidade de uso sustentável. As unidades de proteção integral são subdivididas em estação ecológica; reserva biológica; parque nacional; monumento natural; e refúgio de vida silvestre. Enquanto as unidades de uso sustentável são subdivididas em área de proteção ambiental; área de relevante interesse ecológico; floresta nacional; reserva extrativista; reserva de fauna; reserva de desenvolvimento sustentável; e reserva particular do patrimônio natural.

O Sistema Estadual de Unidades de Conservação de Mato Grosso, (SEUC), classifica as unidades de conservação estaduais e municipais de forma diferente do SNUC. As unidades de proteção integral são subdivididas em estação ecológica, reserva biológica, parques estaduais e municipais, monumento natural e refúgio da vida silvestre. As unidades de uso sustentável são classificadas como área de proteção ambiental, reserva 
extrativista, reserva de desenvolvimento sustentável e estrada parque.

As informações foram obtidas durante o mês de março de 2016. O banco de dados foi descrito quantitativa e qualitativamente, comparado os mesmos dados em diferentes fontes (SEMA-MT e MMA), determinando o número de Uc's, por categorias de uso, extensão, existência de plano de manejo e conselho gestor, entre outras informações.

\section{Resultados e discussão}

As unidades de conservação estaduais possuem valor superior, tanto em extensão quanto em número, quando comparado com as unidades de instância federal no estado de Mato Grosso. Existem casos de arredondamento

Tabela 1 - Unidades de Conservação Federais Mato Grosso

\begin{tabular}{|c|c|c|}
\hline Unidades & № de UC's & 23 \\
\hline \multirow{3}{*}{ Bioma } & Amazônia & 9 \\
\hline & Cerrado & 9 \\
\hline & Pantanal & 5 \\
\hline \multirow{5}{*}{$\begin{array}{c}\text { Proteção } \\
\text { Integral }\end{array}$} & Estação Ecológica & 3 \\
\hline & Reserva Biológica & 0 \\
\hline & Parque & 4 \\
\hline & Monumento Natural & 0 \\
\hline & Refúgio da Vida Silvestre & 0 \\
\hline \multirow{7}{*}{$\begin{array}{c}\text { Uso } \\
\text { Sustentável }\end{array}$} & $\begin{array}{l}\text { Area de Proteção } \\
\text { Ambiental }\end{array}$ & 1 \\
\hline & $\begin{array}{l}\text { Área de Relevante } \\
\text { Interesse Ecológico }\end{array}$ & 0 \\
\hline & Floresta Nacional & 0 \\
\hline & Reserva Extrativista & 0 \\
\hline & Reserva de Fauna & 0 \\
\hline & $\begin{array}{c}\text { Reserva de } \\
\text { Desenvolvimento } \\
\text { Sustentável }\end{array}$ & 0 \\
\hline & $\begin{array}{c}\text { Reserva Particular do } \\
\text { Patrimônio Natural }\end{array}$ & 15 \\
\hline \multirow[b]{2}{*}{$\begin{array}{l}\text { Plano de } \\
\text { Manejo }\end{array}$} & Possui Plano de Manejo & 6 \\
\hline & $\begin{array}{c}\text { Não Possui Plano de } \\
\text { Manejo }\end{array}$ & 17 \\
\hline \multirow[b]{2}{*}{$\begin{array}{l}\text { Conselho } \\
\text { Gestor }\end{array}$} & Possui Conselho Gestor & 10 \\
\hline & $\begin{array}{c}\text { Não Possui Conselho } \\
\text { Gestor }\end{array}$ & 13 \\
\hline \multirow{3}{*}{ Extensão } & Área total SEMA (ha) & $3.779 .881,9$ \\
\hline & Área total MMA (ha) & $3.846 .103,0$ \\
\hline & Diferença (ha) & $66.221,1$ \\
\hline
\end{tabular}

da área de determinadas unidades, na plataforma da SEMA - MT e na do MMA. Para as unidades federais, a diferença no somatório de ambos os órgãos supera os 66.000 ha, correspondendo a duas vezes a área do Parque Nacional de Chapada dos Guimarães (Tabela 1).

Segundo o Art. 2 da Lei 9.985 de 2000 o plano de manejo é conceituado como:

[...] documento técnico mediante o qual, com fundamento nos objetivos gerais de uma unidade de conservação, se estabelece o seu zoneamento e as normas que devem presidir o uso da área e o manejo dos recursos naturais, inclusive a implantação das estruturas físicas necessárias à gestão da unidade.

O Art. $27 \S 3^{\circ}$ da supracitada Lei estabelece: “O Plano de Manejo de uma unidade de conservação deve ser elaborado no prazo de cinco anos a partir da data de sua criação".

Tabela 2 - Unidades de Conservação Estaduais Mato Grosso

\begin{tabular}{|c|c|c|}
\hline Unidades & № de UC's & 46 \\
\hline \multirow{3}{*}{ Bioma } & Amazônia & 16 \\
\hline & Cerrado & 24 \\
\hline & Pantanal & 6 \\
\hline \multirow{5}{*}{$\begin{array}{l}\text { Proteção } \\
\text { Integral }\end{array}$} & Estação Ecológica & 5 \\
\hline & Reserva Biológica & 0 \\
\hline & Parque & 6 \\
\hline & Monumento Natural & 1 \\
\hline & Refúgio da Vida Silvestre & 0 \\
\hline \multirow{5}{*}{$\begin{array}{c}\text { Uso } \\
\text { Sustentável }\end{array}$} & Área de Proteção Ambiental & 6 \\
\hline & Reserva Extrativista & 1 \\
\hline & $\begin{array}{c}\text { Reserva de Desenvolvimento } \\
\text { Sustentável }\end{array}$ & 0 \\
\hline & $\begin{array}{c}\text { Reserva Particular do } \\
\text { Patrimônio Natural }\end{array}$ & 15 \\
\hline & Estrada Parque & 5 \\
\hline \multirow{2}{*}{$\begin{array}{l}\text { Plano de } \\
\text { Manejo }\end{array}$} & Possui Plano de Manejo & 15 \\
\hline & Não Possui Plano de Manejo & 31 \\
\hline \multirow{2}{*}{$\begin{array}{l}\text { Conselho } \\
\text { Gestor }\end{array}$} & Possui Conselho Gestor & 12 \\
\hline & Não Possui Conselho Gestor & 34 \\
\hline \multirow[b]{2}{*}{$\begin{array}{c}\text { UC's Não } \\
\text { Contempladas }\end{array}$} & SEMA & 0 \\
\hline & MMA & 3 \\
\hline
\end{tabular}

Das unidades sem plano de manejo, todas ultrapassaram o prazo máximo legal para elaboração do documento técnico, descumprindo a lei do Sistema Nacional de Unidade de Conservação, porém segundo os relatórios do Ministério do Meio Ambiente, todas se encontram 
em conformidade com o SNUC. O mesmo ocorre com as UC's estaduais (Tabela 2).

O SNUC não prevê Estradas Parque como um tipo de UC, porém, o Estado de Mato Grosso, com o Sistema Estadual de Unidades de Conservação de Mato Grosso (SEUC) cria este tipo de unidade (MATO GROSSO, 1997). O SEUC não apresenta a modalidade Reserva Particular do Patrimônio Natural, porém 15 unidades dessa categoria estão cadastradas no MMA.

No total, não consta no portal do ICMBIO 14 unidades federais. No sistema de relatórios do MMA este valor é ainda maior, com 11 unidades estaduais e 35 unidades municipais não contempladas. A SEMA, órgão executor, responsável pelas UC's estaduais, divulga dados correspondente a todas as instâncias, com apenas uma unidade municipal sem informação, o Parque Natural Municipal Parque Florestal de Sinop.

Das Unidades de Conservação Municipais, 37 no total, apenas 3 possuem informações no MMA disponíveis para os usuários (Tabela 3).

Tabela 3 - Unidades de Conservação Municipais Mato Grosso

\begin{tabular}{|c|c|c|}
\hline Unidades & № de UC's & 37 \\
\hline \multirow{3}{*}{ Bioma } & Amazônia & 7 \\
\hline & Cerrado & 30 \\
\hline & Pantanal & 0 \\
\hline \multirow{5}{*}{$\begin{array}{l}\text { Proteção } \\
\text { Integral }\end{array}$} & Estação Ecológica & 1 \\
\hline & Reserva Biológica & 0 \\
\hline & Parque & 16 \\
\hline & Monumento Natural & 2 \\
\hline & Refúgio da Vida Silvestre & 0 \\
\hline \multirow{5}{*}{$\begin{array}{c}\text { Uso } \\
\text { Sustentável }\end{array}$} & Área de Proteção Ambiental & 17 \\
\hline & Reserva Extrativista & 0 \\
\hline & $\begin{array}{c}\text { Reserva de Desenvolvimento } \\
\text { Sustentável }\end{array}$ & 0 \\
\hline & $\begin{array}{c}\text { Reserva Particular do } \\
\text { Patrimônio Natural }\end{array}$ & 0 \\
\hline & Parque $^{*}$ & 1 \\
\hline \multirow{3}{*}{$\begin{array}{l}\text { Plano de } \\
\text { Manejo }\end{array}$} & Possui Plano de Manejo & 0 \\
\hline & Não Possui Plano de Manejo & 3 \\
\hline & Sem Informações (MMA) & 34 \\
\hline \multirow{3}{*}{$\begin{array}{l}\text { Conselho } \\
\text { Gestor }\end{array}$} & Possui Conselho Gestor & 0 \\
\hline & Não Possui Conselho Gestor & 3 \\
\hline & Sem Informações (MMA) & 34 \\
\hline \multirow{2}{*}{$\begin{array}{c}\text { UC's Não } \\
\text { Contempladas }\end{array}$} & SEMA & 1 \\
\hline & MMA & 34 \\
\hline
\end{tabular}

O SEUC não estabelece a modalidade Parque para UC's de uso sustentável, porém o Parque Natural Municipal Parque Florestal De Sinop, o qual não consta informações na SEMA, é classificado como Parque no MMA.

\section{Conclusões}

Devido à falta de comunicação e repasse de informações entre as diferentes instâncias do Poder Executivo e das próprias unidades de conservação, é possível observar a ineficiência desta rede de gestão. O ICMBIO, responsável por apresentar dados relacionados às UCs federais, não contemplando 14 unidades, e o MMA que deveria compilar todas as unidades, nas três instâncias, não apresenta dados de 46 unidades. Porém, mesmo com várias unidades sem o plano de manejo, em descumprimento com a Lei 9.985/2000, chegando próximo de uma gestão nula, não significa que a as mesmas não estejam cumprindo o objetivo proposto em sua criação.

Uma área protegida gera inúmeros benefícios, indiferentemente da existência do plano de manejo ou do conselho gestor, que facilitam e garantem com eficiência o cumprimento do objetivo da Unidade de Conservação.

\section{Referências}

ARAUJO, H. R.; ÁVILA, G. C.; PAULA, V. C.; SOUZA, D. E. Monitoramento da visitação do Parque Estadual do Biribiri e suas contribuições para o planejamento e gestão do uso público da Unidade de Conservação. Revista Brasileira de Ecoturismo, São Paulo, v.4, n.4, p. 553, 2011.

BELLINASSI, S.; PAVÃO, A. C.; CARDOSO-LEITE, E. Gestão e Uso Público de Unidades de Conservação: um olhar sobre os desafios e possibilidades. Revista Brasileira de Ecoturismo, São Paulo, v.4, n.2, pp.274-293, 2011.

BRASIL, Lei no 11.516 , de 28 de agosto de 2007. Dispõe sobre a criação do Instituto Chico Mendes de Conservação da Biodiversidade - Instituto Chico Mendes; altera as Leis nºs 7.735, de 22 de fevereiro de 1989,11. 284, de 2 de março de 2006, 9.985, de 18 de julho de 2000,10.410, de 11 de janeiro de 2002,11.156, de 29 de julho de 2005,11.357, de 19 de outubro de 2006, e7.957, de 20 de dezembro de 1989; revoga dispositivos da lei no 8.028, de 12 de abril de 1990, e da medida provisória n⿳⺈ 2.216-37, de 31 de agosto de 2001; e dá outras providências. Diário Oficial da União de 29/08/2007, P. 1.

BRASIL. Decreto no 4.340, de 22 de agosto de 2002 . Regulamenta artigos da Lei no 9.985, de 18 de julho de 2000, que dispõe sobre o Sistema Nacional de Unidades de Conservação da Natureza - SNUC, e dá outras providências. Diário Oficial da União de 23/08/2002, P. 9

BRASIL. Lei no 9.985, de 18 de julho de 2000. Regulamenta o art. 225, par. $1^{\circ}$, incisos I, II, III e VII da Constituição Federal, institui o Sistema Nacional de Unidades de Conservação da natureza e dá outras providências. Diário Oficial da União de 19/07/2000, P. 1. 
MATO GROSSO. Lei no 9.502, de 14 de janeiro de 2011. Institui o Sistema Estadual de Unidades de Conservação - SEUC, e dá outras providências. Diário Oficial de $10 / 02 / 2011$.

MEDEIROS, R; PEREIRA, G, S. Evolução e implementação dos planos de manejo em parques nacionais no estado do Rio de Janeiro. Rev. Árvore, Viçosa, v. 35, n. 2, p. 279-288, 2011.

Secretaria de Meio Ambiente de Mato Grosso (SEMA), Unidades de Conservação. Disponível em: <http:// www.sema.mt.gov.br/index.php?option $=\mathrm{com}_{-}$ content\&view $=$ article\&id $=53 \&$ Itemid $=99>$. Acesso em 20 de mar 2016.
TEIXEIRA, C. O desenvolvimento sustentável em unidade de conservação: a "naturalização" do social. Rev. bras. Ci. Soc., São Paulo, v. 20, n. 59, p. 51-66, Oct. 2005.

TERBORGH, J.; SCHAIK, C. V. Por que o Mundo Necessidade de Parques? Tornando os Parques Eficientes Estratégias para a Conservação da Natureza nos Trópicos. Editora UFPR/FBPN. 2002. 